\title{
Geometro-Stochastic Quantization of Gauge Fields in Curved Space-Time.
}

\author{
E. PrugovečKI \\ Department of Mathematics, University of Toronto - Toronto, Canada M5S 1A
}

(Nuovo Cimento A, 100, 827 (1988))

PACS 11.10 - Field theory.

PACS 99.10 - Errata.

The use of $v$-dependent polarization frames $\left(\Lambda_{v} \varepsilon^{(\alpha)}\right)^{*}(k)$ in the geometro-stochastic treatment of photon propagation gives rise to physical states which satisfy the covariant Gupta-Bleuler subsidiary condition (3.7) only for values of $\zeta=(q, v)$ which in a given frame correspond to parallel spatial components of $v$ and $k$. Since the validity of this condition for all $v$ is essential in deriving the action-based expressions (3.37), (3.38) for the GuptaBleuler gauge frame propagator, the $v$-dependence of polarization frames has to be removed from the pertinent formulae (2.8), (2.17), (2.29), (A.4), (A.9), (A.18) and (A.21), thus reverting to the treatment of geometro-stochastic photon propagation originally presented in subsect. 55 of ref. $\left.{ }^{(}\right)$. We thank S. Warlow for pointing out the aforementioned incompatibility.

The corresponding correct formulae for the graviton case, including those for the renormalization constants in (1.3), (A.22) and (A.25), can be found in the paper: $\mathrm{E}$. PRUGOVEČKI: Generally covariant geometro-stochastic quantum gravity, to appear in Nuovo Cimento A, 102 (1989). This paper also supplants ref. $\left({ }^{26}\right)$ on p. 854, which has appeared in Found. Phys. Lett., 2, 81, 163 (1989).

On p. $830 Z\left(\boldsymbol{l}_{0}\right)^{-1}$ should replace $Z\left(\boldsymbol{l}_{0}\right)^{2}$ on the right-hand side of (1.6) and the term between the square brackets in (1.7) should be $i\left(q-q^{\prime}\right)+l_{0}\left(p+p^{\prime}\right)$.

On the right-hand side of (2.23) on p. 835 the superscript $\alpha$ should be $\alpha^{\prime \prime}$.

In eq. (4.20) on p. 848 the term $(-1 / 2) c_{a}(\zeta)$ should be $(-1 / 2) \bar{c}_{a}(\zeta)$.

In the fifth line from the top of p. 853 the expression «algebra-differential forms» should be changed to "algebra related to differential forms".

On pp. 859-861 the term $\left[2\left(\boldsymbol{k}^{2}+m^{2}\right)\right]^{-1 / 2}$ in (A.3a), (A.4), (A.6) and (A.13) should be $\left[2\left(\boldsymbol{k}^{2}+m^{2}\right)^{1 / 2}\right]^{-1}$, and in (A.16) the equality $=1$ should be deleted.

On p. 862 in the integrand of (A.22) $|\boldsymbol{k}|$ should be replaced by $\left(\boldsymbol{k}^{2}+m^{2}\right)^{1 / 2}$.

On p. 864 the subscript $\nu_{\mu}$ on the left-hand side of (B.10) should be $n_{\mu}$. 